\title{
Brillouin Neutron Spectroscopy as a Probe to Investigate Collective Density Fluctuations in Biomolecules Hydration Water
}

\author{
D. Russo, ${ }^{1,2}$ A. Orecchini, ${ }^{1,2,3}$ A. De Francesco, ${ }^{1,2}$ F. Formisano, ${ }^{1,2}$ A. Laloni, ${ }^{1,2}$ \\ C. Petrillo, ${ }^{1,3}$ and F. Sacchetti ${ }^{1,3}$ \\ ${ }^{1}$ CNR-IOM, 34149 Trieste, Italy \\ ${ }^{2}$ Institut Laue-Langevin, 6 rue J. Horowitz, BP 156, 38042 Grenoble, France \\ ${ }^{3}$ Dipartimento di Fisica, Università di Perugia, 06123 Perugia, Italy
}

Correspondence should be addressed to D. Russo, russo@ill.fr

Copyright (C) 2012 D. Russo et al. This is an open access article distributed under the Creative Commons Attribution License, which permits unrestricted use, distribution, and reproduction in any medium, provided the original work is properly cited.

\begin{abstract}
The role of water in the behaviour of biomolecules is well recognized. The coupling of motions between water and biomolecules has been studied in a wide time scale for the self part while collective dynamics is still quite unexplored. Self-dynamics provides information about the diffusion processes of water molecules and relaxation processes of the protein structure. Collective density fluctuations might provide important insight on the transmission of information possibly correlated to biological functionality. The idea that hydration water layers surrounding a biological molecule show a self-dynamical signature that differs appreciably from that of bulk water, in analogy with glass-former systems, is quite accepted. In the same picture Brillouin terahertz spectroscopy has been used to directly probe collective dynamics of hydration water molecules around biosystems, showing a weaker coupling and a more bulklike behaviour. We will discuss results of collective modes of hydration water, arising from neutron Brillouin spectroscopy, in the context of biomolecules-solvent interaction.
\end{abstract}

Keywords: Water dynamics, protein dynamics, hydrophobic interface, collective dynamics, neutron scattering, brillouin spectroscopy

\section{Introduction}

Water, which is the most common molecule in the universe, is one of the most studied simple compounds. In life science it plays a basic role as it represents approximately $70 \%$ of living cell content. In a variety of situations, not only do water molecules determine the structure and dynamics of biomolecules but most biological functions would not take place in their absence [2, 3]. For example, depending on the local environment, water molecules can form a bridge between adjacent sites fixing an ideal conformation, constitute small pools in hydrophobic regions, hydrate specific chemical groups, activate collective motions, or simply constitute the confined liquid medium. Water molecules in the vicinity of a biomolecule may be classified in three categories: internal water, hydration water, and free water. In principle, the internal water and hydration water are more important for protein stability 
and function; however in real living systems also the free water has a relevant role, for instance, allowing for the components transport [4].

In biophysics the description of water dynamics represents a major challenge particularly because it encompasses wide time domains, from picoseconds to milliseconds or more, in an extreme variety of situations, often depending on local conditions and external fields such as temperature and pressure. In this complex scenario, the general emerging picture of hydration water dynamics suggests that the interaction with a biomolecule surface produces, on one hand, a remarkable slowing down of local diffusion dynamics (self) [5-13] and, on the other hand, a sizeable reduction of the propagation lengths of coherent collective modes $[1,14,15]$. All these dynamical aspects are probably relevant in determining the biological functionality.

Among the experimental techniques, nuclear magnetic resonance, light scattering, X-ray, and neutron scattering are the most currently employed to tackle biophysical problems in particular to investigate the hydration water dynamics.

Neutron scattering is a particularly non invasive probe to investigate and determine the structure and dynamics of biological systems and solvent molecules therein. Furthermore, neutron scattering is sensible to the interactions with light atoms like hydrogen, which are uniformly and abundantly distributed in biological matter, being complementary in this respect with X-ray scattering techniques. Neutron spectroscopy provides important information at short time scales, although, in the reciprocal space, the interpretation of the data in complex situations may be difficult because of the different contributions arising from atoms in different local environments. However, being the neutron scattering cross-section strongly dependent on isotopes, exploitation of deuteration, heavy atoms isotope substitution, and matching contrast technique, increases the chance to emphasize the scattering signal from the selected parts of a biological system, thus offering the opportunity to concentrate investigations on one moiety at once of a complex system $[16,17]$.

Many elegant studies, arising from neutron scattering spectroscopy, about the self-dynamics of protein and water molecules at the vicinity of biological molecules exist in the literature [5-8, 18-20]. However, the interest in collective modes, which started to develop with Brillouin spectroscopy at $\mathrm{THz}$ frequencies [21-24], as far as hydration water is concerned, is now taking off with the new possibilities offered by the conception of the dedicated inelastic neutron spectrometer BRISP [25].

The Brillouin spectrometer BRISP is an instrument optimized for small-angle inelastic scattering of thermal neutrons, envisaged to explore collective excitations in a series of different systems. In the last years, it has been extensively employed to tackle the collective dynamical behaviour of water molecules, particularly, in the case of hydration water of biological macromolecules and confined water.

In this paper we will discuss recent results of hydration water collective mode obtaind by neutron Brillouin spectroscopy, in the context of biomolecules-solvent interactions, providing examples of biological samples spanning from small peptides to whole cells.

\section{BRISP: Scientific Background and Instrument General Characteristic}

The new time-of-flight (ToF) Brillouin spectrometer (BRISP) for inelastic neutron scattering has been designed and built at ILL's High Flux Reactor (Figure 1) (A detailed description of the instrument and terms to have access to the instrument, in order to perform experiments, is available on the BRISP web page (http://www.ill.eu/instruments-support/instruments-groups/instruments/brisp/)) [25]. 


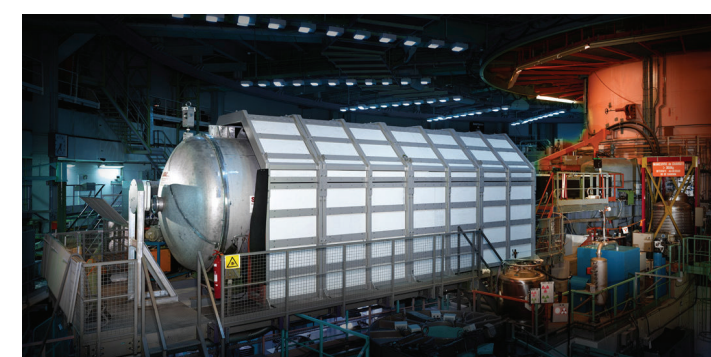

Figure 1: BRISP spectrometer located in the reactor hall of the Institut Laue Langevin. The neutron beam is coming from the right side of the figure where the reactor core is located.

Figure 1 shows the spectrometer inside the reactor hall of the ILL. The spectrometer exploits the timeof-flight [26] concept to perform neutron inelastic scattering experiments over a wide energy range (up to more than $5 \mathrm{THz}$, in both energy loss and energy gain, depending on the momentum transfer) and at low momentum transfer $Q$ (down to $0.1 \mathrm{~A}^{-1}$ in the quasielastic region and up to $1.5 \mathrm{~A}^{-1}$ in the inelastic regime, depending on the energy transfer).

Neutron Brillouin scattering explores a complementary kinematic range compared to Brillouin light scattering, which spans a very small wave vector region due to the long wavelength of the incident radiation, and it is also fully complementary to inelastic X-ray scattering, which provides a specific sensibility to heavy atoms (e.g., $\mathrm{C}$ and $\mathrm{O}$ ) and almost no sensibility to the light hydrogen atoms.

The motivations for accessing this kinematic region, with a clean probe as that represented by inelastic neutron scattering, relate to the understanding of energy transport properties, which can be a microscopic mechanism responsible for the propagation of information across the biomolecule. In particular, BRISP has been designed to allow the investigation of collective excitations characterized by a high propagation speed (sound speed up to more than $3000 \mathrm{~m} \mathrm{~s}^{-1}$ ). This time-of-flight instrument is then aimed at providing the scientific community with a new and efficient tool for advanced investigations of the low-momentum dynamics $f$ of disordered matter ranging from liquids and glasses to compressed gases and biological systems.

An inelastic neutron scattering experiment provides information on the microscopic dynamics through the dynamic structure factor, which is proportional to the probability for an incident neutron to be scattered by the sample with a momentum transfer $\hbar \mathbf{Q}$ and an energy transfer $E$ [26]. A typical experiment on the BRISP spectrometer will give access to a dynamic structure factor $S(Q, \omega)$ in a $(Q, \omega)$ region where the contribution of the coherent part, arising from interparticle correlations, is particularly interesting (Figure 2) [1].

Due to the large incoherent scattering cross-section of hydrogen (related to self-dynamics), with respect to the mainly coherent cross-section of deuterium, a neutron spectroscopy investigation on the coherent dynamics of hydration water can be performed on a sample composed of a completely hydrogenated protein and heavy water [26]. The incoherent signal from the hydrogenated protein will be accounted into the quasielastic peak due to the rather broad energy resolution of BRISP, while the coherent signal, which is produced mainly by the deuterated hydration water, will dominate the purely inelastic spectra. However, in some cases the availability of a fully deuterated sample in heavy water will provide a cleaner separation between biomolecule and hydration water contribution. 


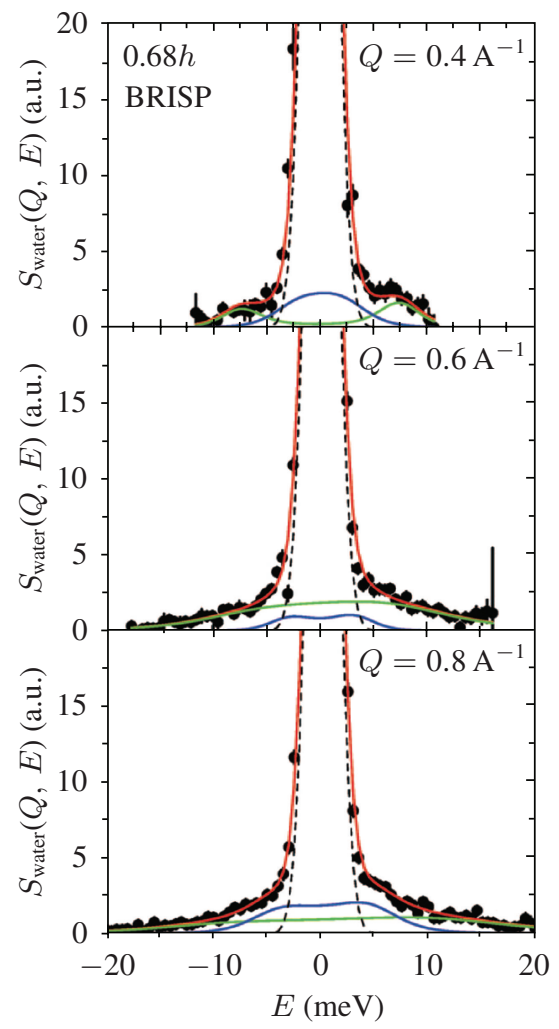

Figure 2: Neutron Brillouin spectrum of protein hydration water, for a protein hydrated at $68 \%$, at different $Q$ values: (a) $0.4 \mathrm{~A}^{-1}$, (b) $0.6 \mathrm{~A}^{-1}$, and (c) $0.8 \mathrm{~A}^{-1}$. The data (dots) are compared with the bestfit function (red full line). The components describing the high- (green line) and low-frequency (blue line) DHO are also shown. The adopted resolution is represented by the dashed line (figure extracted from [1]).

The choice of the model adopted to interpret the $S(Q, \omega)$ depends on physical considerations about the system under investigation. Therefore, the collected spectra, of biomolecules hydration water, are often analyzed using damped harmonic oscillators (DHOs) in order to characterize the water collective excitations propagating throughout the system $[1,14,15]$. This choice is dictated by the need of using the simplest empiric form containing the most important features of a vibrational mode. More complex models can be considered but at the expense of employing a larger number of free parameters. The dependence on the momentum transfer of the proper frequencies, of the DHOs, defines the "dispersion curves," providing a picture of the physical characteristics of active collective motions (optic-like, acoustic-like, dispersive). On the other hand the analysis of the damping factor will give information on the "lifetimes" or propagation lengths, of those motions, somehow related to the local structure of the propagating medium.

In hydrated biological systems, the collective excitations were found to be characterized by the propagation of two modes in analogy with the findings in bulk water: a high-energy linearly dispersing 
mode and a low-energy mode of rather constant energy. In this picture, the hydration water dynamic structure factor can be expressed as follows:

$$
\begin{aligned}
& s(Q, \omega) \\
& \quad=a(Q) \delta(Q)+[n(\omega)+1]\left\{\frac{a_{H}(Q) \Gamma_{H}(Q) \omega}{\left(\omega^{2}-\Omega_{H}^{2}(Q)\right)^{2}+\left(\Gamma_{H}(Q) \omega\right)^{2}}+\frac{a_{L}(Q) \Gamma_{L}(Q) \omega}{\left(\omega^{2}-\Omega_{L}^{2}(Q)\right)^{2}+\left(\Gamma_{L}(Q) \omega\right)^{2}}\right\} .
\end{aligned}
$$

The first term is a Dirac delta function $\delta(\omega)$ of intensity $a(Q)$, which represents both the elastic and the quasielastic responses of the biosystem. The quasielastic component is assumed to have a negligible width as compared to the instrument energy resolution. When this is not the case a finite width function is employed instead of the delta function, (e.g., a Lorentzian function being often fairly appropriate). The term $n(E)$ is the Bose factor, while the term between curly brackets is the sum of two DHO response functions: a high-energy one, whose parameters are identified by the subscript $H$, and a low-energy one, identified by the subscript $L$. Each DHO function is characterized by three $Q$-dependent parameters, that is, the proper frequency $\Omega(Q)$, the damping factor $\Gamma(Q)$, and the intensity $a(Q)$. Examples of experimental data and best fits are represented in Figure 2.

\section{Examples of Collective Dynamics of Biomolecules Hydration Water}

In order to give a snapshot of the instrument potentiality for biology applications, few highlights of BRISP outcome experiments will be described in the following paragraphs. Examples of few dispersion curves and damping factors from hydration water of biological samples, spanning from small peptides to whole cells, will be discussed.

\subsection{Protein Hydration Water}

The determination of $\mathrm{THz}$ dispersion curves for the hydration shell of a biomolecule has been first defined for the ribonuclease A (RNase) protein [1,14] at hydration levels $h$ between 0.7 and $1.0 \mathrm{~g}$ of water per $g$ of protein. The emerging picture indicates that, despite the interaction with the surface of the biomolecule and the slowing down of the translational single particle dynamics, the high frequency collective dynamics of hydration water resembles that of bulk water.

As for bulk water [27-30], in RNase hydration water two collective modes of different characteristics were observed: a high-frequency mode of dispersive nature and a nondispersive lowfrequency mode. The same results were found by MD simulation [31].

In particular, the so-called low-frequency mode displays an optic-like character with a rather constant energy of about 6-7 meV, while the so-called high-frequency mode is instead considered of acoustic-like nature and is characterised by linearly increasing energies with increasing wavevector (left panels of Figure 3). The low- $Q$ slope of this mode provides a propagation speed of about $3400 \mathrm{~m} / \mathrm{s}$; an analogous value was inferred in the case of bulk liquid water. Interestingly, the low-frequency (below $1 \mathrm{GHz}$ ) modes propagate in water, and also in proteins, at a much lower velocity. This effect seems to be a common feature in all biosystems explored to date. On the other hand, the low-frequency mode can 


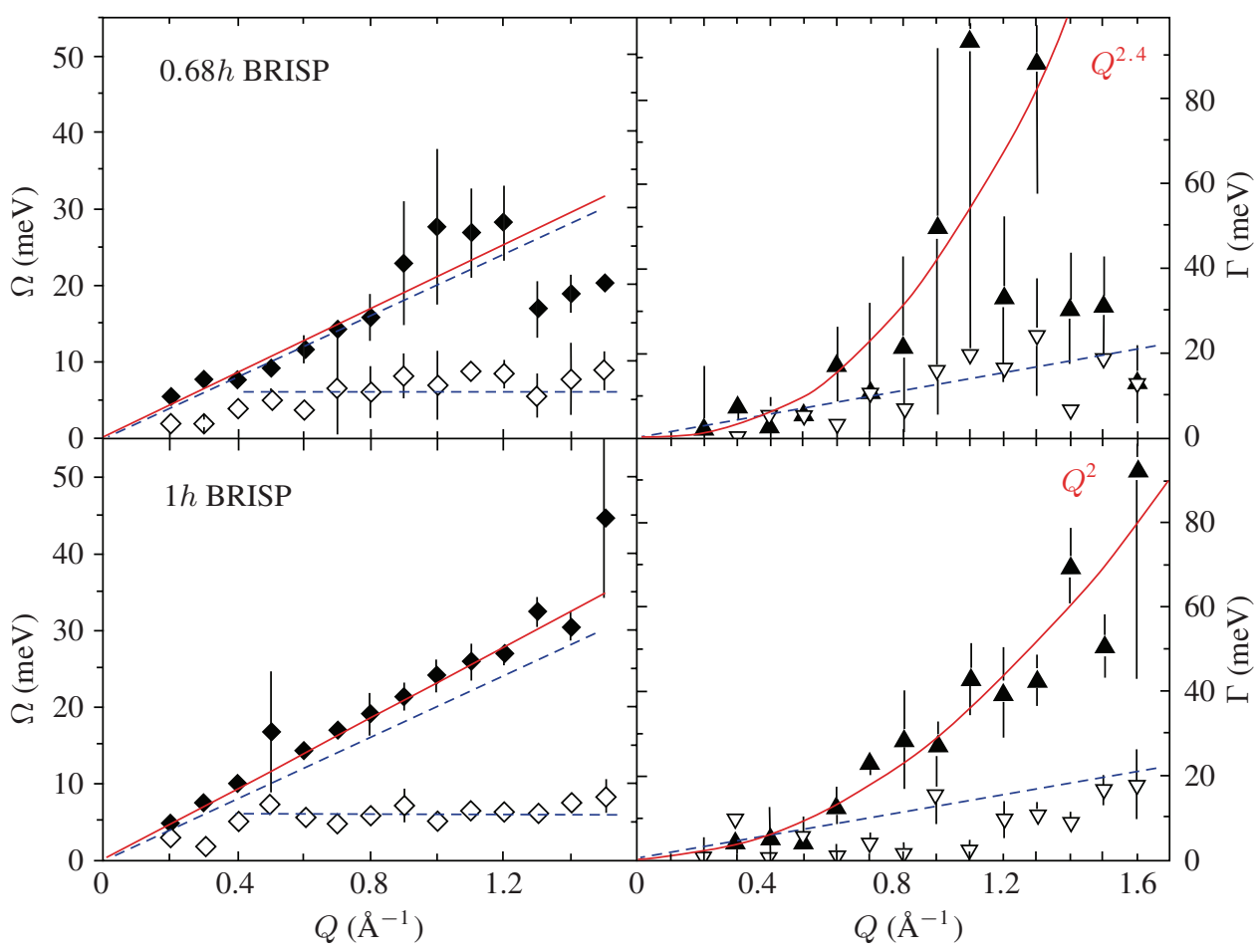

Figure 3: Left panels: dispersion curves of the high-frequency (full diamonds) and low-frequency (empty diamonds) model in RNase hydration water. The red lines represent the linear fit yielding a propagation speed of about $3400 \mathrm{~m} / \mathrm{s}$, and the blue lines report the corresponding curves in pure bulk water. Right panels: damping factors of the high- (full triangle) and low- (empty triangle) frequency mode. The red lines describe the $Q$-dependence of the damping factor as compared to the blue lines representing the bulk water behaviour (Figure extracted from [1]).

be related to $\mathrm{O}-\mathrm{O}-\mathrm{O}$ intermolecular bending motions of the hydrogen bond network [32] or coupling between longitudinal and transverse dynamics [33].

However, different than bulk water behaviour, both excitations were found strongly damped. The high-frequency mode, in particular, shows a $Q^{2}$ increase of the damping factor (right panels of Figure 3). This quadratic dependence reveals that excitations can propagate through a smaller free path than in bulk water at relatively small $Q$. In particular, the density fluctuations become overdamped at $Q$ in excess of about $0.8 \AA^{-1}$, and consequently its propagation becomes more and more hindered and short-ranged. This behaviour can be related to the assumption that the hydration water hydrogen bonding network (HB) is influenced and distorted, compared to its bulk tetrahedral arrangement, by the presence of the biomolecule interface. In particular, on this topic, it was recently suggested that the local "density" of water around biohydrophilic substrates is higher than around biohydrophobic ones [34]. In the case of a whole protein, this will result in an averaged distortion that will appear with an averaged life time of the collective excitations.

With this picture in mind, we can consider the protein molecule as a perturbing probe of an aqueous solvent, capable of introducing novelties in the normally well-defined and ordered structure of 
the water molecules and its hydrogen bond network. In this respect, it becomes naturally interesting to study this behaviour of hydration water in the presence of a different perturbing molecule and check if and how the water structure is affected.

\subsection{DNA Hydration Water and Ionic Solutions}

The coherent density fluctuations propagating through DNA hydration water, $h$ level between 1 and $15 \mathrm{~g}$ of water per $g$ of DNA, were also investigated. Because of the presence of polar groups, the electrostatic interactions are stronger than in the presence of a protein; therefore the HB network of hydration water is expected to be affected in a different way. Indeed, the inferred results were found to be very similar to the globular protein. The two-mode dispersion curves show surprisingly similar characteristic energies $Q$ dependence and damping factors (Figure 4) [15, 35]. The presence in both cases of an excess of modes around 6-7 meV suggests that this energy range may be crucial for hydration water dynamics and its coupling with the biomolecules. In addition, the similarity of hydration water of DNA and protein suggests that the fast collective excitations at the interface with biomolecules are insensitive to the different strength of the solute-solvent interactions. Conversely at larger timescales [ps-ns], a global and stronger slowing down of the dynamics of the first hydration layer is observed [36].

Results from Mamontov and coworkers on ionic solutions, such as lithium chloride aqueous solution $(\mathrm{D} 2 \mathrm{O})_{0.88}(\mathrm{LiCl})_{0.12}$, also suggest that water collective excitations are decoupled from solutesolvent interaction [37]. Also in this particular case, exploring a range of temperature between 270 and $205 \mathrm{~K}$, the presence of a low- and high-frequency excitation clearly appears. The high-frequency acoustic mode shows exactly the same sound velocity probed for the hydration water. However, in opposition to what has been observed around biological systems, the low-frequency mode has a stronger low $Q$ dependence and it allows for the extrapolation of a second propagation speed of $1500 \mathrm{~m} / \mathrm{s}$.

\subsection{Collective Dynamics of Intracellular Water in Living Cell}

To better understand the correlation between the slowing down of diffusive dynamics, hydrogen bond network, and the density fluctuation damping factor, Orecchini and coworkers have investigated the collective dynamics of whole Escherichia coli cells [38].

Water represents approximately $70 \%$ of living cell content, heterogeneously distributed in interstitial, confined and pools of water. In the $E$. coli cells a major fraction of intracellular water shows a diffusive dynamics very similar to that of bulk water. Only approximately a $10 \%$ of the total water was found to be slowed down [39]. In this scenario, the corresponding damping factor is expected to behave differently from that of whole biomolecules.

Results indicate that, although the high-frequency dispersion curves and thus the corresponding propagation velocity resemble those of protein and DNA hydration water, the corresponding damping factors have a clear fingerprint of intracellular water being composed by a considerable amount of free noninteracting water (Figure 5). In particular, the inferred damping factor seems to be a weighted average of damping factors of bulk and hydration water. It turns out that the estimated free-water fraction is about $60 \%$ of the total water and that each molecule is hydrated with about three water layers, a result which indicates a more extended HB network compared to previous examples. 


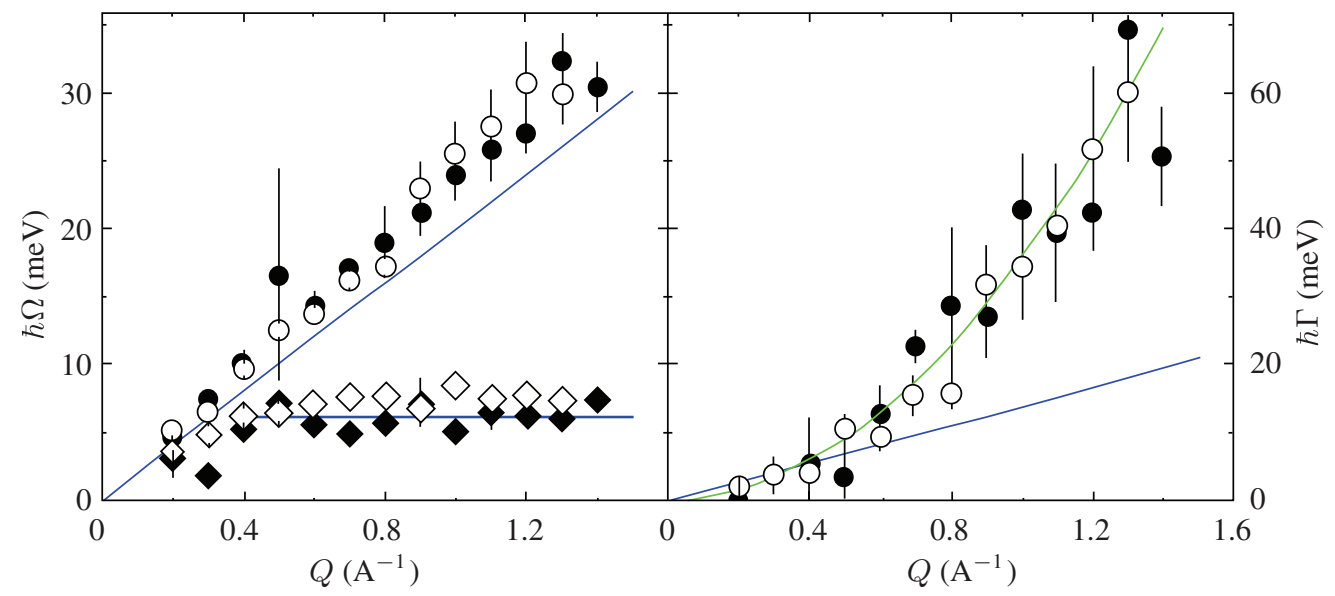

Figure 4: Left panel: experimental dispersion curves in RNase (full symbols) and DNA (empty symbols). Right panel: high-frequency damping factor for RNase and DNA. Dispersion curves and damping factors for bulk water are represented by the blue full lines.

Comparing the low-frequency data to the protein and DNA hydration water, the cellular water seems to have a dependence on $Q$ as the ionic solutions [37]. However, the origin of this second "speed" could be different since in this case the slope does not go naturally through zero. It should be mentioned that the very low-frequency (below $1 \mathrm{GHz}$ ) propagation velocity is much lower than $3000 \mathrm{~m} / \mathrm{s}$ also in DNA and in bacteria, again indicating a common nature of the collective dynamics of water and biologically relevant systems.

From all these examples, it seems then to emerge that the propagation of information through hydration water layer is not affected by the size, the degree of chemical heterogeneity of the bio-surface, and the extension of hydration water HB network. The most surprising effect is the matching of the propagation velocity of the high-frequency sound of hydration water in all the investigated biological systems. Another interesting result that seems to emerge, and that requires a deeper investigation, is that, when a nonnegligible amount of bulk water compared to hydration water is present in the system, the low-frequency mode shows a weak dependence on the momentum transfer. On the other hand, the damping factor seems to bring significant information on the properties of local water structure. This result suggests that the damping has an important role in transferring the density fluctuations from biological molecules to hydration water and vice versa.

\subsection{Hydrophilic and Hydrophobic Peptides Hydration Water}

To shed light at molecular level on the dynamics of hydration shell and control the chemical heterogeneity parameter of the bio surface, Russo and coworkers performed experiments and MD simulation using simplified protein-model biomolecules, with distinct hydrophilic and hydrophobic properties [5, 6, 34, 40, 41]. In a first investigation, the authors have shown that, at room temperature and highly concentrated solution ( $\sim 1$ molecule of solute: 25 water molecules, $\sim 1$ full $4 \AA$ thick hydration layer), the hydration water of hydrophilic (NALMA peptide) and hydrophobic (NAGMA peptide) model 


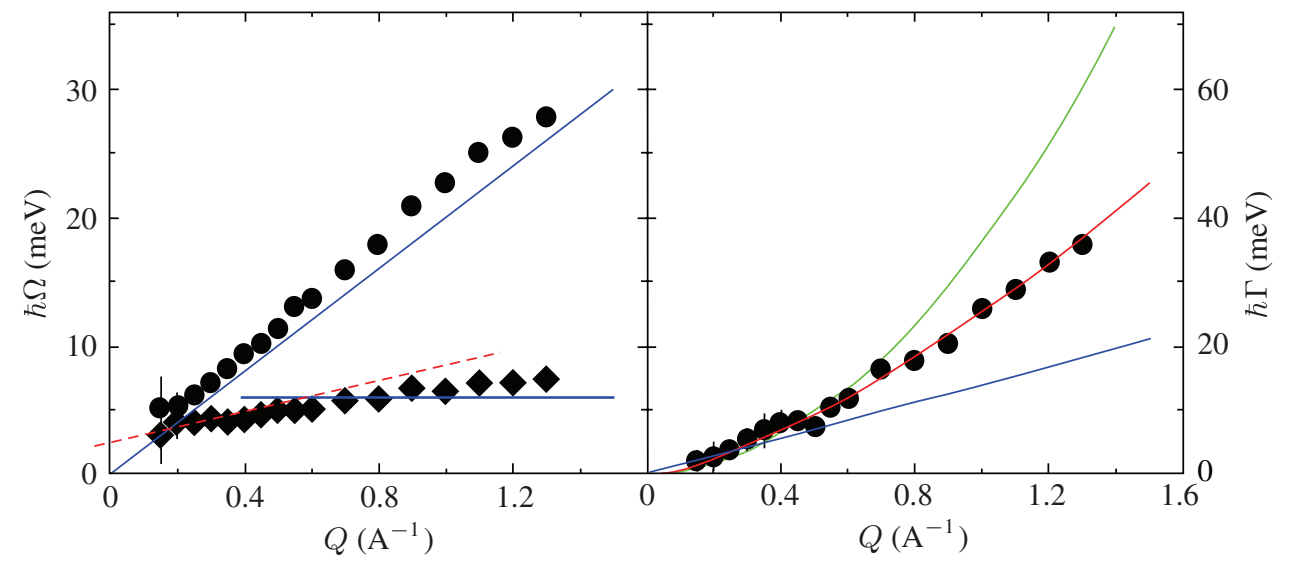

Figure 5: Left panel: experimental dispersion curves of $\mathrm{THz}$ collective modes of $E$. coli intracellular water. Circles refer to high-frequency mode and lozenges to the low-frequency mode. The dashed line is a guide to the eye to follow the low-frequency low- $Q$ dependence. Dispersion curves of bulk water (blue lines) are reported for comparison. Right panel: damping factor of the cellular water (circles) compared to the one of neat water (blue line) and hydration water (Figure extracted from [38]).

peptides shows a translational local diffusion coefficient, resident time, and rotational local diffusion constant that are suppressed with respect to bulk water. It also results that hydration water dynamics is significantly faster around the hydrophilic interface compared to the hydrophobic one, which shows a pronounced anomalous diffusion signature. This different dynamical behaviour is ascribed to a different configuration of the HB network in the first hydration layer for both protein-model biomolecules.

In this framework, measurements of the collective dynamics could provide insight on the specific effect of a hydrophilic and/or hydrophobic interface on the propagation of information at the molecule surface. As for all previously discussed biomolecules, two distinct modes were observed: a dispersive mode and a nondispersive one. The nondispersive mode has been found to be the same for the hydration water layers of both peptide-model systems and it corresponds to an average energy value of 4.5-5 mev. This energy value is distinct from what has been found for neat water and the beforehand discussed macromolecules but it agrees with what was previously observed in the vibrational density of states $[5,6]$. It is also interesting to observe that any slope was detected in the low $Q$ region, as previously observed for the intracellular water and ionic solutions where a nonnegligible amount of bulk water was present.

On the other hand the dispersive modes show two distinct behaviours (Figure 6). The collective density fluctuations propagating in the hydration water surrounding the completely hydrophilic molecule is faster than in the hydrophobic one. The inferred sound speed corresponds to $3600 \mathrm{~m} / \mathrm{s}$ for the hydrophilic case and $\sim 2500 \mathrm{~m} / \mathrm{s}$ for the hydrophobic one. The corresponding damping factor is also higher in the first case, strongly suggesting a different plasticity and structure of the hydrogen bonding network in the two hydration layers. The lower velocity observed in the hydrophobic case, not matching that of the protein and DNA hydration water, suggests that the reduced density and/or the lower interaction of water in the hydrophobic environment have an important effect.

The higher speed probed for the hydrophilic NAGMA hydration layer implies a local environment more dense and/or rigid than the one "generated" from the hydrophobic NALMA. Those preliminary 


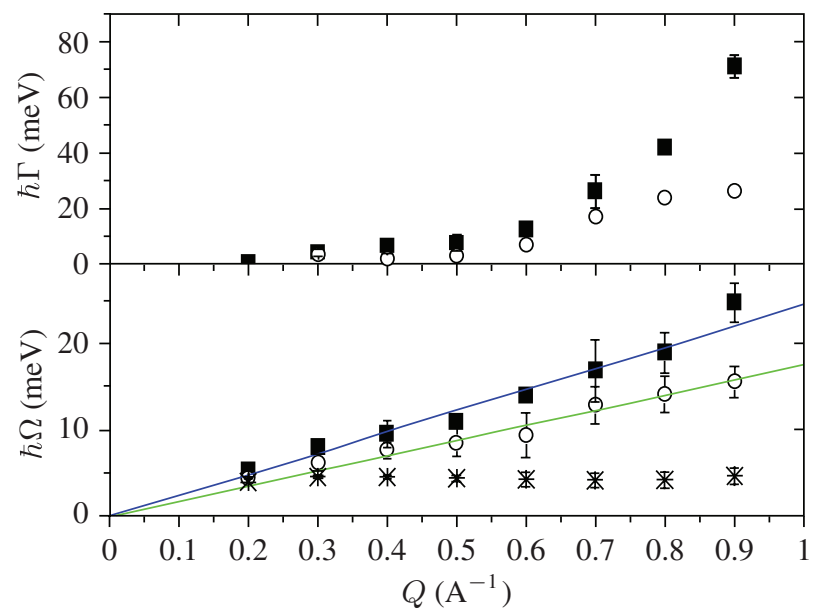

Figure 6: Lower panel: dispersion curves of collective modes of NAGMA and NALMA hydration water. Full squares refer to hydrophilic NAGMA hydration water and empty circles to hydrophobic NALMA hydration water. The green and blue lines correspond to the best fits to infer the propagation speed. The stars report the nondispersive low-frequency mode, which has been probed to be the same for both samples. Upper panel: damping factor for the hydrophilic (full squares) and hydrophobic peptide (empty circles).

results are coherent with what was already proposed by the authors when comparing the vibrational density of states of the two types of protein-model peptides to high-density and low-density amorphous ice $[5,6,34]$.

\section{Conclusions and Perspective}

BRISP represents a unique tool to study the collective properties of aqueous solutions and, in particular, of hydration water. This has opened new perspectives of research in a field which is so far only partially explored. Indeed, first investigations suggest that, even if weaker than the self-dynamics case, water collective dynamics can be modified by the presence of different molecules, including the biological ones, in a nontrivial way. It is too early for driving any major conclusion on the reasons of the different observed behaviour, in particular as far as the nondispersive nature of the low-frequency mode is concerned. However, as a preliminary result one can state that the high-frequency mode propagates at a rather high velocity in all cases, while the interaction of water with a biomolecule affects the damping factor, which is always higher than that of bulk water. The value of the velocity at high frequency is always close to that found in bulk water and, in the same way, also the low-frequency propagation velocity is much lower as it happens in bulk water. This behaviour reminds us of the so-called fast sound, which is observed in water in many thermodynamic conditions.

Finally we observe that these first experiments performed on BRISP have sketched the route to follow: ideal experiments are to be performed on systems where the solute-solvent electrostatic interaction can be freely tuned in a wide thermodynamical (T-P) range. 
In this picture a new field to explore is related to application of high pressure on biological systems. The new interest and demand of the biophysical community, interested in food science and biology under extreme conditions, has pushed to the development and construction of high-pressure sample holder optimized for studying biological solution. The sample holder, made in titanium alloy, reaches pressure up to $30 \mathrm{MPa}$ and will allow investigation of biological molecules under nondenaturting pressure. Preliminary experiments on lysozyme solutions at high concentration show promising results on the importance and the role of water as protecting and plasticizing medium.

\section{Acknowledgments}

The authors are grateful to the ILL for providing neutron beam. D. Russo is thankful to D. Richard for his help in developing and integrating tools, devoted to BRISP data analysis, in the ILL LAMP program.

\section{References}

[1] A. Orecchini, A. Paciaroni, A. De Francesco, C. Petrillo, and F. Sacchetti, "Collective dynamics of protein hydration water by brillouin neutron spectroscopy," Journal of the American Chemical Society, vol. 131, no. 13, pp. 4664-4669, 2009.

[2] G. Careri, E. Gratton, P. H. Yang, and J. A. Rupley, "Correlation of IR spectroscopic, heat capacity, diamagnetic susceptibility and enzymatic measurements on lysozyme powder," Nature, vol. 284, no. 5756, pp. 572-573, 1980.

[3] J. A. Rupley and G. Careri, "Protein hydration and function," Advances in Protein Chemistry, vol. 41, pp. 37-172, 1991.

[4] K. Henzler-Wildman and D. Kern, "Dynamic personalities of proteins," Nature, vol. 450, no. 7172, pp. 964-972, 2007.

[5] D. Russo, J. Copley, J. Ollivier, and J. Teixeira, "On the behaviour of water hydrogen bonds at biomolecular sites: dependences on temperature and on network dimensionality," Journal of Molecular Structure, vol. 972, no. 1-3, pp. 81-86, 2010.

[6] D. Russo, E. Pellegrini, A. G. Miguel, S. Perticaroli, and J. Teixeira, "In situ molecular dynamics analysis of the water hydrogen bond at biomolecular sites: hydrophobicity enhances dynamics heterogeneity," Chemical Physics Letters, vol. 517, no. 1-3, pp. 80-85, 2011.

[7] K. Achterhold, A. Ostermann, M. Moulin, M. Haertlein, T. Unruh, and F. G. Parak, "Dynamical properties of the hydration shell of fully deuterated myoglobin," Physical Review E, vol. 84, no. 4, part 1, Article ID 041930, 2011.

[8] M. C. Bellissent-Funel, J. M. Zanotti, and S. H. Chen, "Slow dynamics of water molecules on the surface of a globular protein," Faraday Discussions, vol. 103, pp. 281-294, 1996.

[9] B. Bagchi, "Water dynamics in the hydration layer around proteins and micelles," Chemical Reviews, vol. 105, no. 9, pp. 3197-3219, 2005.

[10] E. P. Sunde and B. Halle, "Slow internal protein dynamics from water $1 \mathrm{H}$ magnetic relaxation dispersion," Journal of the American Chemical Society, vol. 131, no. 51, pp. 18214-18215, 2009.

[11] N. V. Nucci, M. Pometum, and A. J. Wand, "Mapping the hydration dynamics of ubiquitin," Journal of the American Chemical Society, vol. 133, no. 32, pp. 12326-12329, 2011.

[12] M. Settles and W. Doster, "Anomalous diffusion of adsorbed water: a neutron scattering study of hydrated myoglobin,” Faraday Discussions, vol. 103, pp. 269-279, 1996. 
[13] L. Zhang, L. Wang, Y. T. Kao et al., "Mapping hydration dynamics around a protein surface," Proceedings of the National Academy of Sciences of the United States of America, vol. 104, no. 47, pp. 18461-18466, 2007.

[14] A. Orecchini, A. Paciaroni, A. De Francesco et al., "Brillouin spectroscopy of protein hydration water: new experimental potentialities opened up by the thermal neutron spectrometer BRISP," Measurement Science and Technology, vol. 19, no. 3, Article ID 034026, 2008.

[15] E. Cornicchi, F. Sebastiani, A. De Francesco et al., "Collective density fluctuations of DNA hydration water in the time-window below 1 ps," The Journal of Chemical Physics, vol. 135, no. 2, Article ID 025101, 2011.

[16] G. Zaccai, "Neutrons in biology in the post genome sequencing era," Applied Physics A, vol. 74, supplement 1, pp. S6-S10, 2002.

[17] S. C. M. Teixeira, G. Zaccai, J. Ankner et al., "New sources and instrumentation for neutrons in biology," Chemical Physics, vol. 345, no. 2-3, pp. 133-151, 2008.

[18] D. Russo, J. M. Zanotti, J. Pérez, M. Desmadril, and D. Durand, "Dynamic transition associated with the thermal denaturation of a small beta protein," Biophysical Journal, vol. 83, no. 5, pp. 2792-2800, 2002.

[19] A. Paciaroni, E. Cornicchi, M. Marconi et al., "Coupled relaxations at the protein-water interface in the picosecond time scale," Journal of the Royal Society Interface, vol. 6, supplement 5, pp. S635-S640, 2009.

[20] S. Khodadadi, J. H. Roh, A. Kisliuk et al., "Dynamics of biological macromolecules: not a simple slaving by hydration water," Biophysical Journal, vol. 98, no. 7, pp. 1321-1326, 2010.

[21] M. C. Bellissent-Funel, J. Teixeira, S. H. Chen, B. Dorner, H. D. Middendorf, and H. L. Crespi, "Low-frequency collective modes in dry and hydrated proteins," Biophysical Journal, vol. 56, no. 4, pp. 713-716, 1989.

[22] K. Yoshida, S. Hosokawa, A. Q. R. Baron, and T. Yamaguchi, "Collective dynamics of hydrated $\beta$-lactogloblin by inelastic x-ray scattering," The Journal of Chemical Physics, vol. 133, no. 13, Article ID 134501, 2010.

[23] M. Krisch, A. Mermet, H. Grimm, V. T. Forsyth, and A. Rupprecht, "Phonon dispersion of oriented DNA by inelastic x-ray scattering," Physical Review E, vol. 73, no. 6, Article ID 061909, 2006.

[24] D. Liu, X. Chu, M. Lagi et al., "Studies of phononlike low-energy excitations of protein molecules by inelastic x-ray scattering," Physical Review Letters, vol. 101, no. 13, Article ID 135501, 2008.

[25] D. Aisa, E. Babucci, F. Barocchi et al., "The development of the BRISP spectrometer at the Institut Laue-Langevin," Nuclear Instruments and Methods in Physics Research A, vol. 544, no. 3, pp. 620-642, 2005.

[26] M. Bee, Quasielastic Neutron Scattering, Principles and Applications in Solid State Chemistry, Biology and Materials Science, Adam Hilger, Philadelphia, Pa, USA, 1988.

[27] J. Teixeira, M. C. Bellissent-Funel, S. H. Chen, and B. Dorner, "Observation of new shortwavelength collective excitations in heavy water by coherent inelastic neutron scattering," Physical Review Letters, vol. 54, no. 25, pp. 2681-2683, 1985.

[28] C. Petrillo, F. Sacchetti, B. Dorner, and J.-B. Suck, "High-resolution neutron scattering measurement of the dynamic structure factor of heavy water," Physical Review E, vol. 62, no. 3, pp. 3611-3618, 2000.

[29] F. Sacchetti, J.-B. Suck, C. Petrillo, and B. Dorner, "Brillouin neutron scattering in heavy water: evidence for two-mode collective dynamics," Physical Review E, vol. 69, no. 6, Article ID 061203, 11 pages, 2004.

[30] E. Pontecorvo, M. Krisch, A. Cunsolo et al., "High-frequency longitudinal and transverse dynamics in water," Physical Review E, vol. 71, no. 1, Article ID 011501, 12 pages, 2005. 
[31] M. Tarek and D. J. Tobias, "Single-particle and collective dynamics of protein hydration water: a molecular dynamics study," Physical Review Letters, vol. 89, no. 27, Article ID 275501, 2002.

[32] G. E. Walrafenin, Wat, Wateer; A comprehensive Treatise, vol. 1 of Edited by F. Franks, Pelnum, NewYork, NY, USA, 1972.

[33] A. Cimatoribus, S. Saccani, F. Bencivenga, A. Gessini, M. G. Izzo, and C. Masciovecchio, "The mixed longitudinal-Transverse nature of collective modes in water," New Journal of Physics, vol. 12, Article ID 053008, 2010.

[34] D. Russo, J. Teixeira, L. Kneller et al., "Vibrational density of states of hydration water at biomolecular sites: hydrophobicity promotes low density amorphous ice behavior," Journal of the American Chemical Society, vol. 133, no. 13, pp. 4882-4888, 2011.

[35] A. Orecchini, A. Paciaroni, C. Petrillo, F. Sebastiani, A. De Francesco, and F. Sacchetti, "Water dynamics as affected by interaction with biomolecules and change of thermodynamic state: a neutron scattering study," Journal of Physics, vol. 24, no. 6, Article ID 064105, 2012.

[36] Y. Yonetani and H. Kono, "What determines water-bridge lifetimes at the surface of DNA? Insight from systematic molecular dynamics analysis of water kinetics for various DNA sequences," Biophysical Chemistry, vol. 160, no. 1, pp. 54-61, 2012.

[37] E. Mamontov, A. De Francesco, F. Formisano et al., "Water dynamics in a lithium chloride aqueous solution probed by Brillouin neutron and x-ray scattering," Journal of Physics, vol. 24, no. 6, Article ID 064102, 2012.

[38] A. Orecchini, F. Sebastiani, M. Jasnin et al., "Collective dynamics of intracellular water in living cells," Journal of Physics: Conference Series, vol. 340, Article ID 012091, 2011.

[39] M. Jasnin, A. Stadler, M. Tehei, and G. Zaccai, "Specific cellular water dynamics observed in vivo by neutron scattering and NMR," Physical Chemistry Chemical Physics, vol. 12, no. 35, pp. 10154-10160, 2010.

[40] D. Russo, G. Hura, J. Copley, and T. Head-Gordon, "Hydration dynamics near a model protein surface," Biophysical Journal, vol. 86, no. 3, pp. 1852-1862, 2004.

[41] D. Russo, R. K. Murarka, J. R. D. Copley, and T. Head-Gordon, "Molecular view of water dynamics near model peptides," Journal of Physical Chemistry B, vol. 109, no. 26, pp. 12966-12975, 2005. 


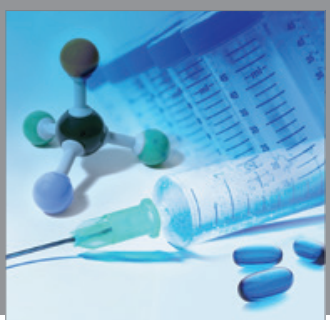

International Journal of

Medicinal Chemistry

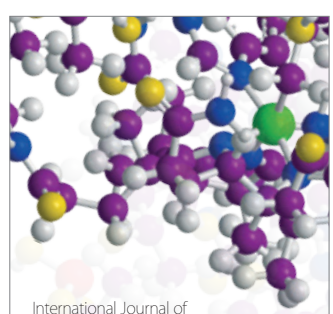

Carbohydrate Chemistry

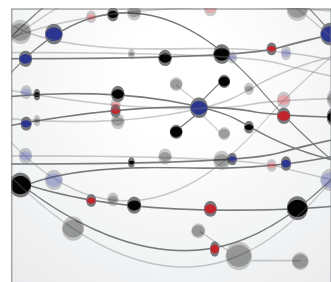

The Scientific World Journal
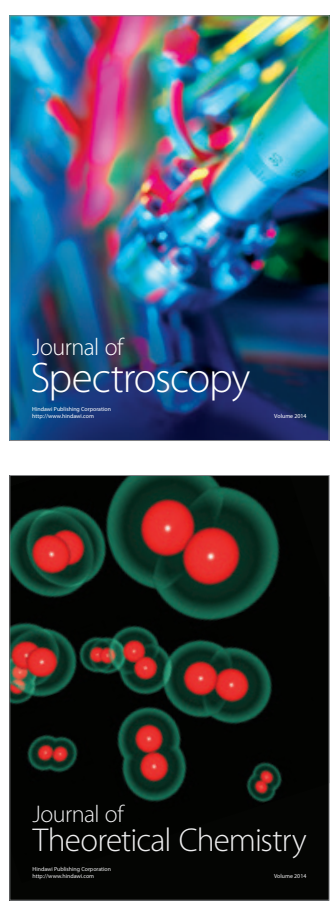
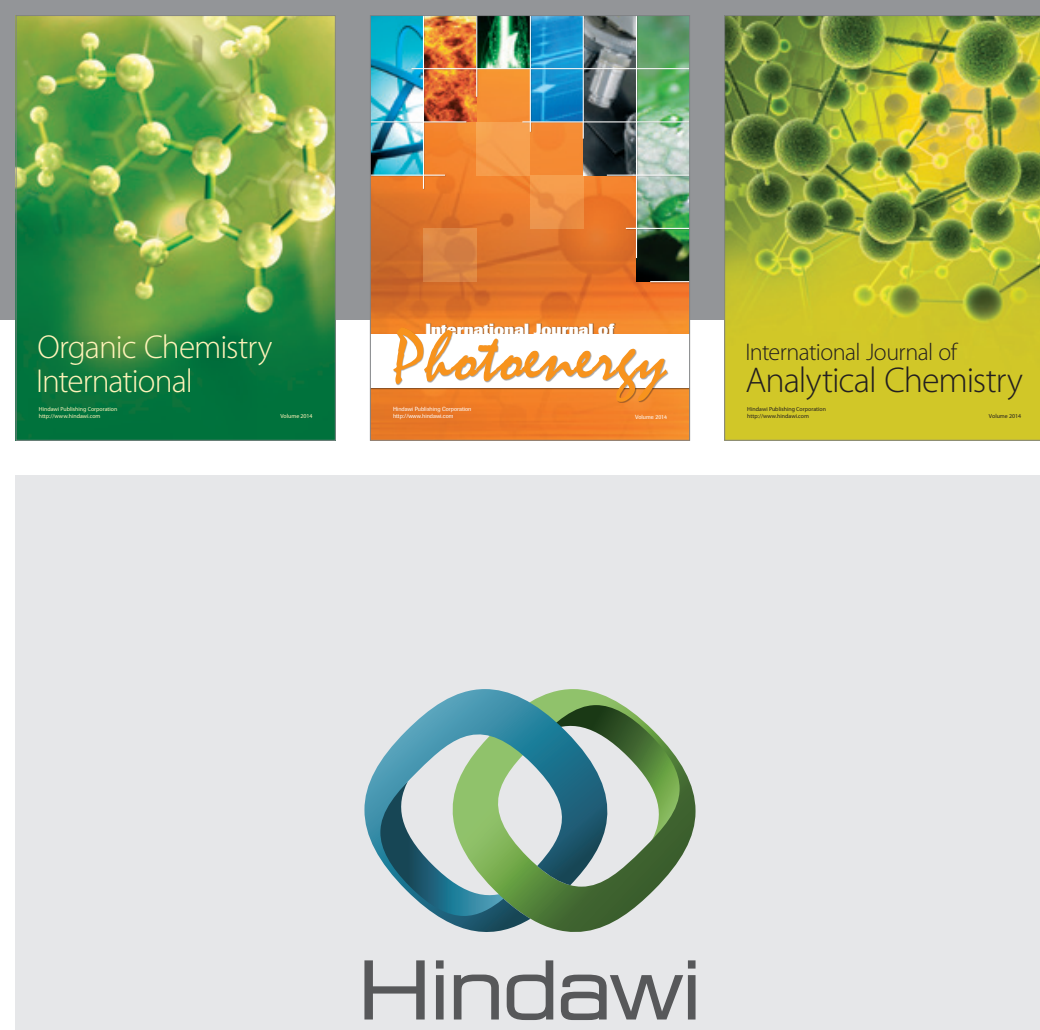

Submit your manuscripts at

http://www.hindawi.com
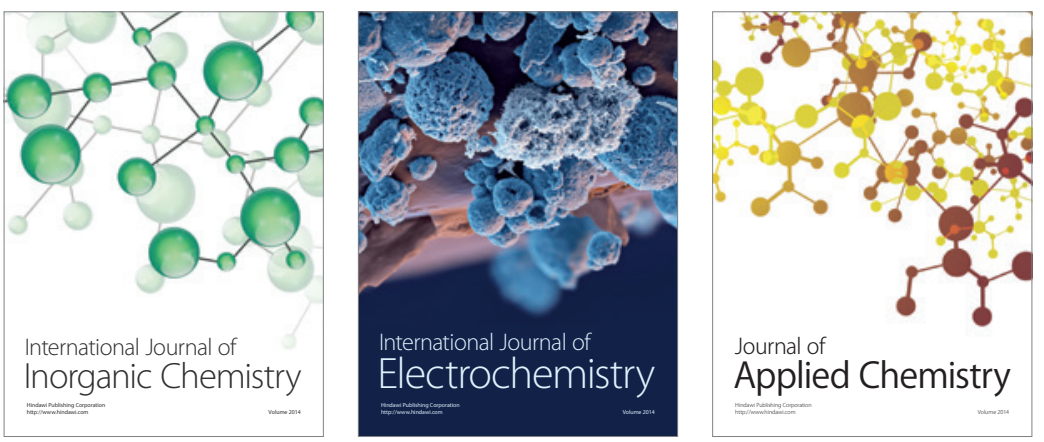

Journal of

Applied Chemistry
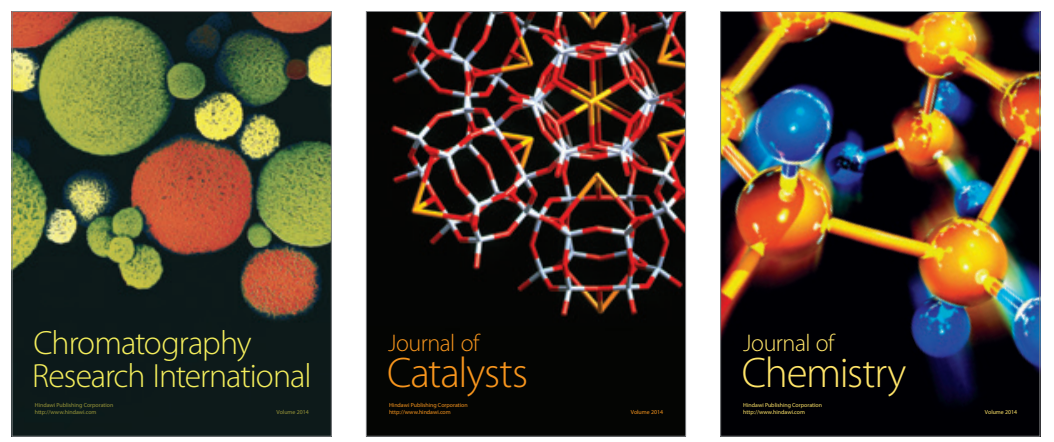
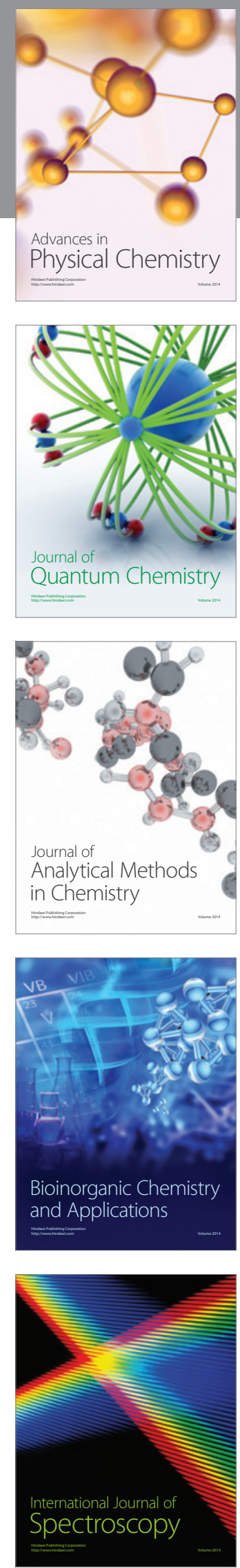